\title{
REFLEXIÓN, OBJETIVACIÓN, TEMATIZACIÓN: SOBRE UNA CRÍTICA HEIDEGGERIANA DE HUSSERL*
}

\author{
Reflection, OBJeCtification, Thematization: \\ on a Heideggerian Critique of Husserl
}

\author{
Felipe León \\ University of Copenhagen - Center for Subjectivity Research \\ Copenhagen, Denmark \\ hbq676@hum.ku.dk
}

\begin{abstract}
Resumen: De acuerdo con una influyente interpretación, ejemplificada por von Herrmann (2000), la postura que se asuma con respecto a la metodología reflexiva marca el contraste entre una vertiente reflexiva y una vertiente hermenéutica de la investigación fenomenológica. Recientemente, autores como Zahavi (2003a, 2005), Crowell (2001) y Cai (2011) han cuestionado la legitimidad de establecer dicho contraste a partir del método de la reflexión. Mi propósito es discutir la crítica central que Heidegger, basándose en Paul Natorp, dirige a la reflexión fenomenológica (GA 56/57, $100)$. Luego de presentar tres posibles interpretaciones de la crítica de Heidegger, y de sugerir la pertinencia de una de ellas, argumento que para contrarrestar dicha crítica es necesario ir más allá de la defensa de la metodología reflexiva que Husserl esboza en el $\S 79$ de Ideas I, y apelar a una distinción entre una objetualidad cósica y una objetualidad temática, sugerida en textos husserlianos posteriores.
\end{abstract}

Palabras clave: reflexión, objetivación, Husserl, Heidegger, epojé fenomenológica.

\begin{abstract}
According to an influential interpretation, epitomized by von Herrmann (2000), the stance that one takes on the reflective methodology determines a contrast between a reflective and a hermeneutic understanding of the phenomenological investigation. In recent years, authors like Zahavi (2003a, 2005), Crowell (2001), and Cai (2011) have challenged the validity of this interpretation. My aim in this article is to discuss the critique that Heidegger, drawing on ideas from Paul Natorp, addresses to phenomenological reflection (GA 56/57, 100). I first present three possible interpretations of Heidegger's critique, and suggest the superiority of one of them. Secondly, I argue that in order to respond to that critique one must go beyond the defense of reflection indicated by Husserl in $\S 79$ of Ideas I, and appeal to a distinction between thing-objecthood and thematic objecthood, suggested in some of Husserl's later writings.
\end{abstract}

Key Words: Reflection, Objectification, Husserl, Heidegger, Phenomenological Epoché.

\footnotetext{
* El presente artículo se enmarca en el proyecto de investigación "Intencionalidad y Trascendencia" (Código DIB: 10983), apoyado financieramente por la División de Investigación de la sede Bogotá (DIB) de la Universidad Nacional de Colombia. Por sus múltiples y muy valiosos comentarios, agradezco a dos evaluadores anónimos de Investigaciones Fenomenológicas, a los profesores Luis Eduardo Gama, Andrés Contreras, Ignacio Ávila, y a los participantes en el X Congreso Internacional de la Sociedad Española De Fenomenología (Barcelona, Noviembre 27-29 de 2013), en el cual fue presentada una versión preliminar de este texto.
} 


\section{INTRODUCCIÓN}

De acuerdo con una influyente interpretación, ejemplificada por FriedrichWilhelm von Herrmann (von Herrmann 2000; cf. Adrián 2005, 2010), la postura que se asuma con respecto a la metodología reflexiva marca el contraste entre una vertiente reflexiva y una vertiente hermenéutica de la fenomenología -la primera desarrollada por Edmund Husserl y la segunda por el joven Martin Heidegger. Recientemente, autores como Dan Zahavi, Steven Crowell y Wenjing Cai han cuestionado la legitimidad de establecer dicho contraste a partir del método de la reflexión (Zahavi 2003, 2005; Crowell 2001; Cai 2011). Mi propósito en este artículo es discutir la crítica central que Heidegger, basándose en Paul Natorp, dirige a la reflexión fenomenológica (Heidegger 2005, 122). Dicha crítica se enfoca en el carácter objetivante de esta última. En primer lugar, ofreceré tres posibles interpretaciones de la crítica de Heidegger, según se entienda ésta como (i) una crítica escéptica, (ii) una irrupción acrítica o (iii) una crítica fenomenológica, y sugeriré la pertinencia de esta última interpretación. En segundo lugar, argumentaré que para contrarrestar la crítica de Heidegger es necesario ir más allá de la defensa de la metodología reflexiva que Husserl esboza en el $\S 79$ de Ideas I, y apelar a una distinción entre una objetualidad cósica y una objetualidad temática, sugerida en textos husserlianos posteriores. Allanar el camino hacia esta distinción requiere, por su parte, reconocer la peculiaridad de la reducción trascendental como método para la investigación sobre la subjetividad.

Husserl y Heidegger difieren en su valoración de la reflexión como método para una investigación fenomenológica sobre la experiencia. Mientras que Husserl afirma que "el método fenomenológico se mueve completamente en actos de la reflexión" (Hua III/1, 144) y que la reflexión es "el título del método de la conciencia para el conocimiento de la conciencia en general" (Hua III/1, 147), la reflexión, según Heidegger, involucra la adopción de una actitud teorética con respecto a las propias experiencias, incapaz de dar razón de éstas de una manera acorde con su modo más original de darse (GA 56/57, 100; cf. Heidegger 2005, 122). En el marco de la filosofía husserliana, las vivencias de reflexión tienen al menos las siguientes cuatro características: (1) estar dirigidas a otra vivencia, (2) ser iterables, (3) fundadas y (4) modificadoras. En primer lugar, las vivencias de reflexión presentan típicamente una estructura dual: en 
ellas una vivencia reflexiva se dirige a una vivencia reflejada. La reflexión, además, se caracteriza por su iterabilidad: dado que no hay una diferencia de naturaleza entre una vivencia reflexiva y una vivencia reflejada - ya que ambas pertenecen a una misma esfera de vivencias - siempre es posible, al menos en principio, ejecutar una reflexión sobre una reflexión (Hua III/1, 147). En tercer lugar, las reflexiones son actos fundados, en la medida en que presuponen un fondo de pre-reflexividad en el que las vivencias se ejecutan conscientemente, aunque sin ser objeto de aprehensiones reflexivas (Hua III/1, 145). Hace parte de la fenomenología de la reflexión que aquello que se tematiza a través de ella se da como algo que ya ha iniciado, esto es, de lo que se era consciente previamente a la ejecución de la reflexión (Hua III/1, 146). Una vivencia prereflexiva, es decir, previa al ser captada por una vivencia reflexiva que la tome por objeto, proporciona un sustrato indispensable para la realización de una reflexión. De lo anterior se desprende una cuarta característica: "cada "reflexión" tiene el carácter de una modificación de conciencia [...]" (Hua III/1, 148. Énfasis en el original). La reflexión no produce arbitrariamente su objeto, sino que se ejerce necesariamente sobre un fondo de pre-reflexividad que, además, se ve modificado por la reflexión (Hua I, 72; cf. Husserl 2005, 76).

A pesar de reconocer su carácter modificador, Husserl no pone en entredicho la pertinencia y centralidad de la metodología reflexiva en su proyecto fenomenológico. Por el contrario, desde su perspectiva, la modificación reflexiva posibilita que el fenomenólogo no se limite a reproducir las vivencias en su inmediatez (Hua I, 72; cf. Husserl 2005, 77). Heidegger, en cambio, considera que la modificación reflexiva conlleva una distorsión del modo más originario como nos están dadas nuestras experiencias. Si el propósito de la fenomenología es tematizar las experiencias en su originariedad, lo cual no equivale a reproducirlas en su mera inmediatez, parecería haber una discrepancia fundamental entre un modo reflexivo de acceder a nuestras experiencias y el modo que tienen estas últimas de manifestarse originariamente en la trama de la vida. La razón es que, de acuerdo con Heidegger, la reflexión fenomenológica implica la adopción de una actitud teorética sobre las experiencias, que las objetiva y les induce lo que en el Kriegnotssemester de 1919 (en adelante KNS) llama una Entlebung, o "privación de vida". Así, en una lección del mismo período Heidegger afirma que "los correlatos de la "intuición inmanente", de la "reflexión" son, en lo fundamental, aprehendidos como cosas" (GA 58, 238. Trad. 
mía. Énfasis en el original). ${ }^{1}$ Por contraste, la meta principal que Heidegger se traza en dicha lección es reconocer que la vida no es algo objetual: tal y como lo expresa "[...] la vida no es un objeto y no puede nunca convertirse en un objeto: no es algo que tenga el carácter de lo objetual [Objektartiges]. Reconocer esto es nuestra meta principal" (GA 58, 236. Trad. mía). ${ }^{2}$

¿Pero habría acaso una manera de acceder a una investigación sobre las experiencias que no involucre la adopción de lo que Heidegger llama una actitud teorética? Parte fundamental de su propuesta consiste en responder afirmativamente a esta pregunta. Aquí me limitaré a destacar algunos elementos de dicha propuesta. Una fenomenología como ciencia originaria (Urwissenschaft) (GA 56/57, 63; cf. Heidegger 2005, 75), que haga justicia a nuestra experiencia pre-teorética del mundo circundante, tendría que dar razón de las relaciones de familiaridad, significatividad y proximidad que entretejemos con un entorno, y, en esa medida, no podría basarse en la perspectiva distanciada y presuntamente neutral típica de la teorización. Vivir en un entorno (Umwelt) significa para Heidegger que todo tiene un primario e irrenunciable carácter significativo, todo, como dice, "mundea" (es weltet) (GA 56/57, 73; cf. Heidegger 2005, 88). En el KNS, Heidegger describe la experiencia del mundo circundante a partir del ejemplo de entrar al aula de clase y ver la cátedra desde la cual el profesor dicta la lección. Lo que destaca en primer lugar con respecto a esta vivencia es que, si nos atenemos sin prejuicios a cómo nos es dada, la vivencia no resulta de la sumatoria de determinadas sensaciones, percepciones de formas, de la percepción de una cosa o de un objeto de uso. En otras palabras, lo que se ve no son fragmentos que se ensamblen progresivamente en la vivencia de ver la cátedra, sino que lo que de entrada se ve es la cátedra desde la que el profesor habla.

Heidegger no pretende negar que mediante un procedimiento abstractivo sea posible, partiendo de la vivencia de ver la cátedra, llegar hasta la sensación de marrón y convertirla en un objeto de consideración. En tal caso, según señala, dicha sensación "se muestra como algo primariamente dado" (GA 56/57, 85; cf. Heidegger 2005, 103). Sin embargo, el costo de que la sensación esté

\footnotetext{
1 "Dagegen werden die Korrelate der "immanenten Anschauung", der "Reflexion" usw. im Grunde als Dinge aufgefasst." (GA 58, 238. Énfasis en el original)

2 "[...] das Leben is kein Objekt und kann nie Objekt werden: es ist nichts Objektartiges. Das zu erkennen ist unser Hauptziel." (GA 58, 236)
} 
dada de este modo es la destrucción del mundo circundante (GA 56/57, 91; cf. Heidegger 2005, 109). Heidegger sostiene que el "verdadero responsable de la deformación de la problemática [del mundo circundante]" es el "dominio general de lo teorético, [el] primado de lo teorético" (GA 56/57, 87; cf. Heidegger 2005, 105) e incluso habla de "una progresiva y destructiva infección teorética de lo circundante", que al propagarse en lo circundante suprime su carácter significativo. Esta propagación de lo teorético tiene un carácter progresivo, esto es, procede gradualmente y por ello mismo es rastreable en las series conceptuales mediante las que se teoriza, por ejemplo "cátedra, caja, color marrón, madera, cosa" (GA 56/57, 89; cf. Heidegger 2005, 107). Así pues, el carácter presuntamente primario de la sensación como objeto sólo se muestra en la medida en que uno se encuentra instalado ya en la actitud teorética, que "sólo es posible cuando se destruye la vivencia del mundo circundante" (GA 56/57, 85; cf. Heidegger 2005, 103). No se trata de dos momentos consecutivos, primero destrucción de esta vivencia y luego asunción de la actitud teorética, sino que una cosa va inextricablemente ligada a la otra. Como indica, "[c]uando intento explicar el mundo circundante en clave teorética, éste se derrumba. El intento de disolver y someter metodológicamente las vivencias a teorías y explicaciones carentes de toda clarificación, no significa una intensificación de las vivencias, no conduce a un mejor conocimiento del mundo circundante" (GA 56/57, 86; cf. Heidegger 2005, 104). Por lo demás, no se trata, para Heidegger, de convertir a la vivencia del mundo circundante en un más allá inaccesible, o en un principio caótico e incomprensible. Como lo aclara en una lección del mismo período, "[I]a vida no es un enredo caótico de oscuros flujos, ni un principio de fuerza sordo, ni un exceso sin límites que lo enreda todo, sino que es lo que es sólo como una forma concreta significativa." (GA 58, 148. Trad. mía). Lo que hay que comprender es la estructuración propia de la vivencia en y desde sí misma, y esto no debería conducir a ningún misticismo o irracionalismo.

Es previsible que la postura heideggeriana frente a la actitud teorética tenga repercusiones sobre su valoración del método reflexivo. En efecto, este último es considerado como un modo paradigmático de actitud teorética:

La mirada reflexiva convierte una vivencia que inicialmente no era observada, que se vivía simplemente de manera arreflexiva [reflexionslos], en una vivencia "observada" [»erblickten«]. La observamos. En la reflexión la tenemos delante de nosotros, estamos dirigidos hacia ella y la convertimos en un objeto sin más [Objekt, Gegenstand überhaupt]. Es decir, en la reflexión adoptamos una actitud teorética. Todo comporta- 
miento teorético, dijimos, es privante de vida [entlebendes]. Esto se muestra en un sentido eminente con respecto a las vivencias. En la reflexión, éstas ya no son vividas, sino observadas -este es el sentido de la reflexión. Colocamos las vivencias delante de nosotros, arrancándolas del vivenciar inmediato; metemos la mano, por así decirlo, en la corriente fluctuante de las vivencias y entresacamos una o varias vivencias, es decir "detenemos la corriente", como dice Natorp [...]. (GA 56/57, 100; cf. Heidegger 2005, 122. Traducción modificada. Énfasis en el original)

Puede que en la reflexión fenomenológica las vivencias que son objetos de consideración no se descompongan en sensaciones simples, como podría ocurrir en la teorización científica, pero esto no quita que dicha reflexión involucra una fisura en la experiencia, un tomar distancia frente a uno mismo, que impediría una aprehensión adecuada y no distorsionante de las propias experiencias. Es lo que Heidegger destaca al retomar las observaciones de Paul Natorp (1912, 190). El momento de objetivación inherente a la reflexión fenomenológica sería un lastre inevitable para esta última.

La crítica heideggeriana a la actitud teorética y, específicamente, a la reflexión fenomenológica suscita varias preguntas, que a continuación me propongo abordar: ¿Cuál es exactamente la naturaleza de la crítica de Heidegger a la objetivación reflexiva? ¿Se trata de una crítica escéptica, que busca cuestionar la validez de hecho de la reflexión? O, por el contrario, ¿tiene la crítica de Heidegger una naturaleza distinta, quizás más radical? ¿Aboga esta crítica por un abandono de la reflexión fenomenológica no por su intrínseca inadecuación para alcanzar lo que promete, sino quizás porque no está ni siquiera en condiciones de prometerlo?

Una primera alternativa consiste en interpretar la crítica de Heidegger como una crítica escéptica. Al señalar que la reflexión involucra una concepción procesual y objetivante de las vivencias, Heidegger estaría poniendo en duda que la reflexión alcance lo que promete. La pregunta crucial, por supuesto, es cómo se alcanzaría el reconocimiento de que las vivencias se presentan como procesos y objetos bajo la mirada reflexiva, ya que éste es el punto clave del cuestionamiento. Supongamos que, con talante husserliano, ejecutamos una reflexión sobre la vivencia de ver la cátedra al entrar al auditorio, con el fin de analizar la estructura noético-noemática de la vivencia. Desde la perspectiva heideggeriana que aquí tomamos en consideración, al reflexionar sobre la vivencia la tomo como objeto, la observo, y con esto la modifico irremediablemente. Incluso si intentara captar la vivencia en su extensión temporal, no pasaría de ser un proceso temporalmente extendido ante mí, un Vor-gang. Las 
experiencias en la reflexión, como Heidegger lo pone perspicuamente, "marchan ante la mirada [vor dem Blick paradieren, vorbei-marschieren]" (GA 58, 123. Trad. mía).

¿Pero cómo se alcanza la convicción de que la vivencia se presenta en la reflexión como un proceso o un objeto? Aquí se presentan al menos dos alternativas: esta convicción se puede alcanzar o bien de modo reflexivo, o bien de un modo no reflexivo. Dado que lo que está en juego es la legitimidad de la convicción misma, tendría que haber necesariamente un suelo de evidencia para suscribirla. La pregunta central es cómo se accede a este suelo. Supongamos, primero, que a este suelo de evidencia no se accede reflexivamente. De acuerdo con una sugerencia contenida en el KNS, y desarrollada por von Herrmann (2000), podemos asumir que para Heidegger la evidencia no es un asunto de reflexión sino de "simpatía con la vida [Lebenssympathie]" (GA 56/57, 110; cf. Heidegger 2005, 133, von Herrmann 2000, 92), es decir, de un sumergirse en el flujo experiencial de la vida en el que ésta busca una autocomprensión en sus propios términos, en su propio lenguaje, al margen de toda teorización (GA $58,42)$. Si optamos por este camino, sin embargo, nos encontramos con una discrepancia fundamental entre el método para alcanzar la convicción antes mencionada y el contenido de la convicción misma. En efecto, ¿acaso es plausible suponer que a través de Lebenssympathie podría alcanzarse la convicción de que las vivencias se presentan en la reflexión de manera procesual y objetual? Si "simpatía con la vida", como señala von Herrmann, es el "Gegenwort zur Reflexion" (2000, 92), no parece plausible sostener que a una convicción acerca de la naturaleza modificadora de la reflexión pueda llegarse siguiendo dicho camino. Por otra parte, por supuesto, podría sostenerse que a la convicción mencionada se llega reflexivamente: es decir, que es sólo a través de una reflexión de segundo grado, dirigida a la reflexión previamente ejecutada sobre la vivencia de ver la cátedra, que puedo llegar a asegurar que esta vivencia se presenta en la reflexión de primer grado como un objeto o un proceso. Si es así, sin embargo, habría que conceder la razón a Husserl, cuando argumenta que la posibilidad de poner en duda a la reflexión necesariamente la presupone (Hua III/1, 154).

Hay que destacar que, a pesar de sus diferencias, estas dos maneras de leer la crítica de Heidegger dan por descontado que ésta es una crítica escéptica. Lo que se pone en cuestión, en ambos casos, es el importe cognoscitivo de la 
reflexión, la capacidad de esta última de capturar adecuadamente la experiencia pre-reflexiva. La estructura de esta crítica podría expresarse, en términos generales, de la siguiente manera: si la reflexión fenomenológica pretende capturar la experiencia pre-reflexiva, no cumple su cometido. Pero hay una segunda y más radical interpretación de la crítica de Heidegger, que consiste en entender esta última como una crítica que emerge plenamente del nivel de la prereflexividad: la reflexión, desde esta perspectiva, implicaría una desnaturalización de las vivencias, porque es una metodología por principio (y no de hecho) inadecuada para tematizarlas. Hay una diferencia importante, sobre la cual quisiera llamar la atención, entre cuestionar la reflexión fenomenológica porque no está en condiciones de cumplir lo que promete o porque no está siquiera en condiciones de prometerlo. A diferencia de la primera interpretación, esta lectura sostiene que la reflexión fenomenológica no puede pretender abordar la experiencia pre-reflexiva, porque reflexión y pre-reflexividad están en niveles incomunicados. Según esta lectura, la pre-reflexividad es una dimensión refractaria a cualquier tipo de teorización $y$, por ende, es en sentido estricto areflexividad. Esta segunda interpretación conlleva una clara ruptura entre la fenomenología reflexiva y la fenomenología pre-reflexiva, y respalda la lectura dicotómica tradicional sobre la relación entre ambas vertientes de la investigación fenomenológica. No obstante, la aparente radicalidad de la crítica tiene un precio a pagar: ¿hasta qué punto seguiría siendo una crítica de la fenomenología reflexiva? Para ponerlo en otros términos: ¿hasta qué punto sería un cuestionamiento capaz de disuadir, o al menos de interpelar fenomenológicamente a los partidarios de la reflexión acerca de la validez de esta última, y no un punto de arranque totalmente distinto de la investigación?

Al parecer, pues, nos encontramos con un dilema al interpretar la crítica heideggeriana de la reflexión fenomenológica: si se toma como una crítica escéptica, parece presuponer a la reflexión misma. Por otra parte, si en lugar de tomarla como una crítica escéptica la concebimos como una ruptura radical, (como una 'crisis', en el sentido etimológico de krinein: separación, división) una bifurcación o un punto de arranque nuevo, no es claro en qué medida seguiría siendo una crítica de la reflexión fenomenológica. Ésta, al parecer, permanecería inmune en sus pretensiones, pues su legitimidad no habría sido puesta en entredicho. Lo que se habría mostrado es que es al menos concebible tomar un camino alternativo en la investigación fenomenológica, pero, incluso 
contando con un desarrollo pormenorizado de esta alternativa, no se habrían ofrecido razones para optar entre uno u otro camino. Mi manera de enfrentar este dilema consiste en cuestionar la plausibilidad de ambos cuernos del dilema - por lo tanto, del dilema mismo-, y exponer una tercera posibilidad

Primero explicaré por qué considero inviables ambos cuernos del dilema. En primer lugar, sería ingenuo concebir la crítica de Heidegger como una crítica escéptica, basada en adoptar una postura epistemológica con respecto a la experiencia pre-reflexiva. Dicha postura es presupuesta incluso por el escéptico que cuestiona la legitimidad cognoscitiva de la reflexión. Husserl discute con un adversario escéptico en el $\S 79$ de Ideas I. Recordemos el núcleo de la crítica de dicho adversario: si el vivenciar inmediato es una "realidad absoluta" vivida (erlebt) como tal, pero no conocida, ¿cómo podrían establecerse afirmaciones con respecto a dicha realidad? En otras palabras, ¿cómo podría conocerse el vivenciar inmediato, si éste por principio repele la objetualidad (o el ser-objeto) requerida por todo conocer? La presuposición del escéptico es aquí que el conocimiento requiere necesariamente de un objeto de conocimiento. Sin embargo, hay razones para suponer que Heidegger no aceptaría esta presuposición. En efecto, su crítica a la actitud teorética es precisamente que dicha actitud se basa en una objetivación de lo que se contempla teoréticamente, en una estructura epistemológica dual, que consta de un sujeto cognoscente y un objeto por conocer. La crítica heideggeriana, sin embargo, no se amolda a esta estructura epistemológica, anclada en la actitud teorética. Si lo hiciera caería en el sinsentido de ser una crítica teorética de lo teorético.

Las anteriores consideraciones parecen sugerir, por descarte, la viabilidad del segundo cuerno del dilema. En efecto, una manera sugestiva y relativamente difundida de interpretar el carácter no epistemológico de la crítica de Heidegger consiste en verla como una ruptura en la base de la investigación fenomenológica. Desde esta perspectiva, la crítica de Heidegger a la fenomenología reflexiva y a la actitud teorética se prevendría por principio de recaer en esta última mediante la adopción y el desarrollo de un punto de arranque nuevo de la investigación: la crítica de Heidegger sería en realidad una irrupción. Podría pensarse que lo que Heidegger pone en marcha en el KNS es algo que ni siquiera ha sido vislumbrado por los partidarios de una fenomenología reflexiva. La esfera de la vida pre-reflexiva, que los partidarios de la reflexión creen poder tematizar, sería, en el marco de este modelo, un dominio que requiere de una 
metodología y una conceptualidad totalmente autónoma, que nada tienen que ver con la reflexión. Así, los partidarios de esta interpretación de la crítica de Heidegger podrían sostener que la primera interpretación de ésta (el primer cuerno del dilema) concede de entrada demasiado: la legitimidad de plantear la crítica en términos de la capacidad de la reflexión para abordar la experiencia pre-reflexiva.

Ya he indicado un primer problema en el que incurre esta lectura: el riesgo de convertir la crítica de Heidegger a la fenomenología reflexiva en una ruptura acrítica con respecto a esta última. En efecto, si dicha 'crítica' tiene el carácter de un nuevo comienzo, el de una irrupción y no el de un cuestionamiento de la estructura misma de la reflexión, no es claro por qué habría que seguir llamándola una crítica. En segundo lugar, esta interpretación no concuerda con clara evidencia textual. En el KNS Heidegger observa que "[s]e ha de romper con esta primacía de lo teorético, pero no con el propósito de proclamar un primado de lo práctico o de introducir otro elemento que muestre los problemas desde una nueva perspectiva, sino porque lo teorético mismo y en cuanto tal remite [zurückweist] a algo pre-teorético" (GA 56/57, 59; cf. Heidegger 2005, 71. Énfasis en el original). Es claro que, para Heidegger, la superación de la actitud teorética no se da asumiendo una nueva perspectiva (ateorética), sino mostrando la fundación de la actitud teorética en una experiencia más originaria (preteorética) (cf. GA 58, 228).

La alternativa que aquí quisiera explorar es que la crítica heideggeriana a la reflexión no es ni una crítica escéptica ni una irrupción acrítica, sino una crítica fenomenológica. Cuando Heidegger cuestiona que las vivencias se presenten como objetos bajo la mirada de la reflexión, su interés no es poner en duda, a la manera de un escéptico, que dicho modo de aparición se ajuste al modo de ser verdadero de las vivencias. Por ponerlo en otros términos, su crítica no se apoya en un contraste entre la apariencia y la realidad de la vida pre-reflexiva, pues dicho contraste estaría anclado en la estructura sujeto-objeto típica de la teorización, que Heidegger se propone superar (cf. GA 63, 81). Pero su crítica tampoco consiste en la irrupción súbita de un nuevo punto de vista, por las razones anteriormente aducidas. La crítica heideggeriana a la reflexión es, más bien, inmanente a la investigación fenomenológica, y allí recae una de sus innegables fortalezas (GA 20, 140; cf. Heidegger 2006, 134). Este carácter fenomenológico de la crítica de Heidegger puede reconocerse en la reapropiación 
heideggeriana del "principio de todos los principios" husserliano, en el marco del KNS. Como he señalado antes, es sobre la base de una objeción de Natorp que Heidegger critica la objetivación inherente a la reflexión fenomenológica (GA 56/57, 101; cf. Heidegger 2005, 122). Sin embargo, las referencias a Natorp por parte de Heidegger en el KNS no terminan aquí. Heidegger también observa que "[...] Natorp, con toda su perspicacia a la hora de plantear el problema [de lo teorético], no ha agotado todas las posibilidades. [...]En su confrontación con la fenomenología no llega a abordar la auténtica esfera de problemas." (GA 56/57, 109; cf. Heidegger 2005, 132).

Resulta decisivo que el concepto de Lebenssympathie, como respuesta a la objeción de Natorp, sea derivado por Heidegger a partir del "principio de todos los principios" de la investigación fenomenológica, que Husserl formula en Ideas I. Heidegger, citando parcialmente a Husserl, menciona este principio de la siguiente manera: "'todo lo que se da originariamente en la "intución" [...] hay que tomarlo simplemente como se da"'" (GA 56/57, 109; cf. Heidegger 2005, 132). No obstante, más adelante en el texto de Heidegger se pone de presente que su interpretación del "principio de todos los principios" husserliano poco tiene que ver con los planteamientos de Husserl mismo: "Se trata de la intención originaria de la auténtica vida, de la actitud originaria del vivir y de la vida cuanto tales, de la absoluta simpatía con la vida que es idéntica con el vivir mismo" (GA 56/57, 110. Énfasis en el original). Unas páginas después observa: "La vivencia que se apropia de lo vivido es la intuición comprensiva, la intuición hermenéutica, la formación originariamente fenomenológica que vuelve hacia atrás mediante retroconceptos y que se anticipa con ayuda de preconceptos y de la que queda excluida toda posición teorético-objetivante y trascendente" (GA 56/57, 117; cf. Heidegger 2005, 142. Énfasis en el original).

Estas observaciones heideggerianas, cuyo análisis desborda los propósitos del presente artículo, ponen de presente que a través de los conceptos de simpatía con la vida e intuición hermenéutica, Heidegger cuestiona implícitamente el marco reflexivo de la fenomenología husserliana, aunque, al mismo tiempo, la manera como Heidegger llega a dichos conceptos es fenomenológica. Lo anterior sugiere que el estrato más básico del debate metodológico entre una fenomenología reflexiva y una fenomenología hermenéutica, es una divergencia sobre el carácter objetivante de la intuición fenomenológica, o, como lo expresa Heidegger, sobre la cuestión de si el correlato de la intuición es algo Objektarti- 
ges (cf. GA 58, 237). Los partidarios de la reflexión no pueden defender que la intuición originaria y dadora a la que se refiere el "principio de todos los principios" es de naturaleza reflexiva apelando a un principio fenomenológico más básico, porque, recordemos, aquél es el "principio de todos los principios". Pero, exactamente por la misma razón, los detractores de la reflexión no pueden conmover a un nivel más básico la posición de base de sus adversarios.

\section{Algunas estrategias para Replicar a la Crítica de Heidegger}

Una primera estrategia para replicar a la crítica de Heidegger consiste en señalar que el concepto fenomenológico de reflexión es polisémico. Dan Zahavi (2003a, 2005) ha argumentado que Husserl, Sartre y Merleau-Ponty distinguen varios tipos de reflexión fenomenológica. Con respecto a Husserl, observa que en Zur Phänomenologie des inneren Zeitbewusstseins este último escribe que las experiencias reflejadas adquieren un nuevo modo de ser, al ser acentuadas (herausgehoben), y que esta acentuación no es más que su ser aprehendidas. Husserl también describe a la reflexión como un ejercicio de articulación y explicitación de los componentes y estructuras ya implícitos en la experiencia prereflexiva. ${ }^{3}$ Merleau-Ponty, por su parte, sugiere que la reflexión fenomenológica no conlleva una distorsión sino una consumación de la experiencia (Zahavi 2005, 89), mientras que Sartre plantea una importante distinción entre una "reflexión pura" y una "reflexión impura": mientras que la segunda reifica la experiencia y opera con una dualidad epistémica, la primera es descrita como una tematización no distorsionante de la experiencia pre-reflexiva (Zahavi 2005, 89).

Con base en estas ideas, Zahavi plantea una distinción entre dos tipos fundamentales de reflexión fenomenológica: una reflexión objetivante (objectifying) y una reflexión atencional (attentional). Desde su perspectiva, ambas involucran una modificación de la experiencia pre-reflexiva $(2005,88)$. No obstante, mientras que la reflexión objetivante toma a la experiencia pre-reflexiva como un objeto e implica una fisión o fragmentación en la experiencia, la re-

\footnotetext{
3 "[Husserl] wrote that the experience to which we turn attentively in reflection acquires a new mode of being; it becomes accentuated (herausgehoben). He argued that this accentuation is nothing other than its being grasped (Hua 10/129). Husserl also spoke of reflection as a process that discloses, disentangles, explicates, and articulates all those components and structures that were implicitly contained in the pre-reflective experience (Hua 24/244; 11/205, 236)." (Zahavi 2005, 88)
} 
flexión atencional es un tipo de reflexión que se limita a acentuar y destacar, en lugar de objetivar. Se trata de una forma peculiar de "estar despierto" ("wakefulness"), o, siguiendo a Fink, de un "schauendes Hinnehmen" que articula las experiencias con particular intensidad en lugar de objetivarlas (Zahavi 2005, 88. Énfasis en el original). No obstante, Zahavi también provee razones para no equiparar irrestrictamente la reflexión con una modificación atencional. Por ejemplo, según la caracterización husserliana estándar de la reflexión, ésta involucra una vivencia reflexiva y una vivencia reflejada, mientras que la atención, según Husserl, es una característica de un acto primario, y no un nuevo acto (Zahavi 2005, 88). En todo caso, el carácter polisémico del concepto de reflexión fenomenológica lleva a Zahavi a afirmar lo siguiente: "Heidegger distingue entre una reflexión objetivante, por una parte, y, por otra, una tematización y articulación hermenéutica no objetivante. Otros fenomenólogos harían básicamente la misma distinción, pero insistirían en que se trata de una distinción entre dos tipos de reflexión, un tipo distorsionante y objectivante, por una parte, y un tipo de reflexión no objetivante, que se limita a acentuar. En mi opinión, es razonable afirmar que lo que Heidegger ha hecho realmente es describir en detalle el segundo tipo de reflexión" (Zahavi 2005, 96. Trad. mía). ${ }^{4}$

Una segunda estrategia para cuestionar la lectura dicotómica de la relación entre la fenomenología reflexiva y la fenomenología hermenéutica ha sido desarrollada por Steven Crowell (2001), quien ha argumentado que la fenomenología hermenéutica del joven Heidegger, en lugar de ser una antítesis, involucra una reapropiación del concepto husserliano de reflexión. Crowell sostiene que una lectura atenta de las lecciones tempranas de Heidegger en Friburgo muestra que la reflexión "no es abandonada, sino reinscrita (sin ser mencionada) en un planteamiento de la filosofía como un modo específico de comportamiento interrogativo" (Crowell 2001, 131. Trad. mía. Énfasis en el original). ${ }^{5}$. Para Crowell, el rasgo metodológicamente esencial de la reflexión es que "con el fin de llevar a cabo un cuestionamiento filosófico sobre la base de evidencia auténtica, tengo que atender explícitamente, al mismo tiempo, a mi propio "acto" de

\footnotetext{
4 "Heidegger distinguishes between an objectifying reflection and a non-objectifying hermeneutical thematization and articulation. A number of other phenomenologists would basically make the same distinction, but would insist that it is a distinction between two different types of reflection, a distorting and objectifying type and a non-objectifying and merely accentuating type. In my view, it is reasonable to claim that what Heidegger has really done is to describe the latter in detail." (Zahavi 2005, 96)

5 " [Reflexion] is not abandoned but reinscribed (without being denoted) into an account of philosophy as a distinctive sort of questionning comportment." (Crowell 2001,131. Énfasis en el original)
} 
cuestionar" (Crowell 2001, 144. Trad. mía). ${ }^{6}$ La manera como Crowell entiende el carácter reflexivo de la fenomenología para el joven Heidegger depende de concebirla como una actividad esencialmente interrogativa, que involucra inevitablemente a quien interroga: "la definición plena de la filosofía implica un momento de reflexión, dado que el ente que filosofa está concernido por su propio ser en cuanto ser" (Crowell 2001, 143. Trad. mía). ${ }^{7}$

Una tercera estrategia para cuestionar la lectura dicotómica antes mencionada ha sido propuesta recientemente por Cai (2011). Según Cai, el método hermenéutico heideggeriano "ofrece un modelo de le reflexión que no es carente de mundo, sino situado y con su propio horizonte por iluminar" (Cai 2011, 10. Trad. mía). ${ }^{8}$ En particular, la fenomenología hermenéutica contribuiría a una noción más enriquecida de reflexión fenomenológica, al destacar el carácter situado de ésta, como una "reflexión-en-el-mundo". Retomando una descripción de Gadamer, Cai destaca la incompletitud ("unfinishedness (Unvollendbarkeit)" (Cai 2011, 83)) de toda reflexión fenomenológica, concebida como un cuestionamiento progresivo del horizonte en el que tiene lugar, y que nunca puede traer totalmente a la luz. La interpretación de Cai sobre la relación entre la fenomenología reflexiva y la fenomenología hermenéutica es, como las lecturas de Zahavi y Crowell, compatibilista, con la particularidad de que su estrategia para mostrar la compatibilidad entre ambas es un esfuerzo por mostrar la complementariedad de ambas, construyendo una noción de "reflexión hermenéutica [hermeneutic reflection]" que combine elementos de una y otra. En cierto sentido, la manera como Cai construye el claro contraste inicial entre la reflexión trascendental husserliana y la hermenéutica heideggeriana facilita la tarea de mostrar la complementariedad de ambas bajo la noción de reflexión hermenéutica. Sin embargo, hay dos reparos básicos que pueden plantearse a su línea de argumentación.

En primer lugar, un presupuesto central del argumento de Cai es que la reflexión trascendental husserliana es "carente de mundo [worldless]" (Cai 2011, 81. Trad. mía). Al emplear el término "worldless" aplicado a la reflexión tras-

\footnotetext{
6 " $[\mathrm{I}] \mathrm{n}$ order to accomplish philosophical questioning on the basis of authentic evidence I must at the same time attend explicitly to my own "act" of questioning." (Crowell 2001, 144)

7 " $[T]$ he full definition of philosophy implicates a moment of reflection, since the being who philosophizes must concern itself with its own being as being." (Crowell 2001,143)

8 "[Heidegger's hermeneutical method] offers a model of reflection which is not worldless but situated and has its own unilluminated horizon." (Cai 2011, 10)
} 
cendental, Cai pretende Ilamar la atención sobre el hecho de que "el espectador fenomenológico no está situado más en un mundo familiar, compuesto de habitualidades incuestionadas, tradiciones, etc. La ingenuidad de tener un mundo como horizonte es, para Husserl, la primera cosa que el fenomenólogo necesita interrumpir" (Cai 2011, 81). ${ }^{9}$ Lo que parece encontrarse tras esta interpretación de Cai es una lectura particular de la epojé y la reducción fenomenológicas. Cai asume que la actitud fenomenológica (trascendental) implica dejar atrás la familiaridad con el mundo y el concebirlo como un horizonte experiencial. Sin embargo, aunque resulta plausible suponer que la actitud fenomenológica conlleva dejar atrás una consideración ingenua de la familiaridad con el mundo y del horizonte mundano, resulta menos plausible asumir que dicha actitud conlleva dejar atrás toda consideración del mundo en términos de familiaridad y horizonte. En particular, puede argumentarse que la actitud fenomenológica abre precisamente el espacio para una consideración fenomenológica de estas nociones (cf. Zahavi 2009). Después de todo, Husserl insiste en distintas ocasiones en que la epojé no conlleva una exclusión del mundo, y enfatiza que este último sigue valiendo como tal en la actitud fenomenológica. Lo que la epojé excluye es una cierta actitud ingenua ante el mundo, que consiste en considerarlo como existente independientemente de la conciencia (cf. Zahavi 2003b, 2009).

Un segundo reparo a la lectura de Cai es que oscila entre distintas cuestiones concernientes a la reflexión fenomenológica. Por una parte, está la cuestión metodológica concerniente a cómo acceder a una investigación fenomenológica de la experiencia pre-reflexiva. Por otra parte, está la cuestión ético-existencial acerca del valor que la reflexión puede tener para la vida humana. Con respecto a este punto, Cai sostiene que "la reflexión lleva consigo una esencial dimensión ético-existencial, que es más primaria que su función epistémica" (Cai 2011, 199. Trad. mía). ${ }^{10}$ Al respecto, sin embrago, cabe señalar que incluso si la reflexión tuviera una dimensión ético-existencial, no es claro por qué habría que buscar en esta dimensión los elementos para responder a la cuestión metodológica. Al desarrollar la complementariedad entre la fenomenología reflexi-

\footnotetext{
9 "The phenomenologizing spectator is no longer situated in a familiar world that is comprised of unquestioned habitualities, traditions, etc. The naivety of having a world as the horizon is for Husserl the first thing that he phenomenologist needs to put to an end." (Cai 2011, 81)

10 "Reflexión carries an essential ethical-existential dimensión that is more primary tan its epistemic function" (Cai 2011, 199).
} 
va y la fenomenología hermenéutica, no se resuelve el núcleo de la divergencia metodológica entre ambas, relativo al carácter objetivante de la reflexión.

\section{OBJETUALIDAD TEMÁTICA}

Dejando de lado sus respectivos méritos, las propuestas interpretativas que acabo de mencionar no destacan suficientemente el aspecto estructural que subyace a la crítica heideggeriana. Dicho aspecto apunta a la centralidad que el problema de la objetivación reflexiva tiene en la crítica de Heidegger. Incluso si, siguiendo a Zahavi, aceptamos que la fenomenología hermenéutica se basa en el uso de un tipo de reflexión fenomenológica, que otros fenomenólogos (Husserl incluido) también tematizan, la crítica de Heidegger podría replantearse con respecto a la reflexión objetivante. Por otra parte, si, siguiendo a Crowell, adoptamos una noción general de reflexión como un "comportamiento interrogativo", la discusión podría tornarse fácilmente en un asunto terminológico, relativo a la amplitud de la noción de reflexión con la que uno desea operar. Mientras que el argumento de Zahavi depende, como hemos visto, de distinguir variedades de la reflexión fenomenológica y de caracterizar la contribución de Heidegger como un tipo particular de reflexión, la lectura de Crowell parece incurrir en el problema de operar con muy pocas variedades de reflexión. Los partidarios de la fenomenología hermenéutica o pre-reflexiva podrían, al parecer, adoptar la noción máximamente general de reflexión que Crowell emplea, pero esto no los llevaría a cuestionar la incompatibilidad entre la fenomenología reflexiva y la fenomenología hermenéutica. El concepto estricto de reflexión fenomenológica, como objetivación de las vivencias, parece quedar en la penumbra y, por lo tanto, la crítica de Heidegger sigue en pie para este concepto.

Lo anterior sugiere que el punto crítico de la divergencia no es el concepto mismo de reflexión, sino el carácter objetivante de esta última. ¿En qué sentido es, pues, objetivante la reflexión fenomenológica, o un tipo fundamental de esta última? Heidegger, por supuesto, no critica que en la reflexión fenomenológica las vivencias se conviertan literalmente en cosas. Su observación puntual, recordemos, es que "los correlatos de la "intuición inmanente", de la "reflexión" son en lo fundamental [im Grunde] aprehendidos como cosas" (GA 58, 238. Trad. y énfasis míos). En otras palabras, su crítica no se basa en una equiparación inusitada entre las vivencias reflejadas y las cosas percibidas ex- 
ternamente. Pero lo dado reflexivamente se daría siempre bajo la forma de un objeto que 'hace frente' a un sujeto. En la reflexión las vivencias se presentarían como cosas que marchan ante un observador (GA 58,123). No obstante, el presupuesto de Heidegger puede cuestionarse. En particular, la objetualidad reflexiva no tiene por qué pensarse en los términos en los que Heidegger la describe, y hay varios indicios de que Husserl mismo no lo hace. La razón fundamental, en mi opinión, es que hacerlo implicaría pasar por alto el carácter distintivo de la reflexión trascendental con respecto a la reflexión psicológica.

La reflexión trascendental tematiza a la subjetividad, pero no la convierte en un objeto cósico, sino en un tema de investigación. Gegen-stand, Ding, Objekt, Vor-gang, todos estos términos cruciales en la crítica de Heidegger a la reflexión están enraizados en una comprensión pre-trascendental de la subjetividad, en concebirla como objetividad (de tipo cósico) y no como subjetividad. El punto decisivo radica en reconocer que hay una manera tradicional de abordar la subjetividad que no logra desprenderse de una consideración objetivante (en sentido cósico) de la misma. La reflexión psicológica, o natural, asume íntegramente la tesis de la actitud natural, al considerar como existentes los objetos a los que se dirige - que son, en este caso, experiencias. Como Husserl lo expresa: "Pero así como el yo se encuentra en la reflexión natural como yohombre no sólo referido al mundo, sino a sí mismo como un ente mundano (espacio-temporal), así también encuentra todas sus experiencias experienciantes [erfahrenden Erlebnisse], todo lo subjetivo que se puede encontrar en la reflexión, precisamente como algo mundanamente real" (Hua XXXIV, 226. Cf. Ibid., 93. Trad. mía). ${ }^{11}$ Debido a este compromiso de la reflexión psicológica con lo "mundano-real" es fundamental distinguirla de la reflexión fenomenológico-trascendental, que presupone la ejecución de la epojé (Hua XXXIV, 222223; Husserl 2005, 77). Aprehender la epojé en toda su radicalidad implica aplicarla no sólo con respecto al carácter de existencia del mundo, sino también con respecto a una consideración mundana de la subjetividad, es decir, una que tome a las experiencias subjetivas como ocurrencias espacio-temporales que tienen lugar 'en' el sujeto. Lo anterior sugiere que, en la medida en que la sub-

\footnotetext{
11 "Aber wie das Ich sich in der natürlichen Reflexion als Menschen-Ich und nicht nur weltbezogen, sondern selbst als in der Welt als weltliches Seiendes (raumzeitliches) vorfindet, so auch all seine erfahrenden Erlebnisse, alles in Reflexion vorfindliche Subjetktive, eben auch als weltlich Reales." (Hua XXXIV, 226)
} 
jetividad sea vista como un espacio 'interior' en el que se localizan las experiencias a las que se dirige la reflexión, no hemos abandonado aún el campo de la reflexión psicológica.

Estas consideraciones sugieren la relevancia de distinguir no sólo entre la reflexión psicológica y la fenomenológica, tal y como Husserl lo hace en distintos lugares, sino también entre estos dos tipos de reflexión y la epojé. Ésta última, en efecto, es precondición de la reflexión fenomenológica, y marca con ello la diferencia central entre ésta y la reflexión psicológica. La epojé, por lo tanto, puede interpretarse como un acto sui generis (Hua III/1, 55) que permite neutralizar o poner fuera de juego el carácter de existencia del mundo, los objetos circundantes y de nosotros mismos y nuestras vivencias en tanto que existencias espacio-temporales, y concentrarnos en la dimensión experiencial. Pero el acto mismo de la epojé no corresponde ni a los parámetros de una reflexión psicológica, que está aún basada en la actitud natural, ni a los de una reflexión fenomenológica, que presupone el acceso al campo de investigación abierto precisamente por la epojé.

Hasta ahora me he ocupado de lo que podría llamarse una dilucidación noemática de la intencionalidad reflexiva, y he criticado que el objeto intencional de la reflexión tenga que concebirse en términos cósicos. No obstante, podría pensarse que la objetualidad cósica es ante todo una determinación noética, relativa al acto en el que algo se da como cosa. En otras palabras, podría argüirse que cuando Heidegger critica la objetivación reflexiva, el núcleo de su crítica no está dirigido a que la reflexión cosifique las experiencias, sino a la división o separación intra-experencial que la reflexión traería consigo, al margen del efecto que ésta tenga sobre su objeto intencional. Desde esta perspectiva, la reflexión sería objetivante porque introduciría una fisura, una fragmentación en la experiencia. Esta separación parece manifestarse en la estructura dual que Husserl asigna a la reflexión, en la separación entre un acto aprehensor o reflexivo y un acto aprehendido o reflejado. La estructura estratificada de la reflexión indicaría, pues, una cuestionable auto-fragmentación.

Al respecto, hay que reconocer que muchas descripciones de Husserl motivan la aproximación de la intencionalidad reflexiva a la intencionalidad perceptual. Indudablemente, la metáfora del ver en la reflexión está presente en muchas de sus caracterizaciones de la reflexión. Como Petitmengin y Bitbol han destacado, la caracterización más clara de la reflexión fenomenológica provista 
por Husserl involucra una "auto-fisión [self-fission] entre un sujeto reflejado y un sujeto que reflexiona" (Petitmengin \& Bitbol 2011, 27. Trad. mía). Asimismo, la idea husserliana según la cual el fenomenólogo se convierte en un "espectador desinteresado" (Husserl 2005, 77; Hua VIII, 92; Hua XXXIV, 86) de las vivencias, parece ajustarse a la idea de que la reflexión implica un irrenunciable momento de auto-fragmentación. Sin embargo, como Petitmengin y Bitbol también sugieren, la postura de Husserl acerca de este "dualismo interno [inner dualism]" (Petitmengin \& Bitbol 2011, 27. Trad. mía) implicado por la reflexión es mucho más matizada de lo que algunas afirmaciones sueltas pueden sugerir: "No es verdad, él [Husser] escribe, que al reflexionar sobre un acto de percepción me vuelvo ciego al objeto percibido. De hecho, durante la reflexión 'sigo viendo claramente todo' (Husserl 1959, p. 111)" (Petimengin \& Bitbol 2011, 27). ${ }^{12}$

Cuando Husserl hace la afirmación citada al final de este pasaje, está discutiendo lo que considera una tergiversación de la epojé fenomenológica. Observa que ésta no es una suerte de "auto-hipnosis [Selbsthypnose]" (Hua VIII, 111) al cabo de la cual el fenomenólogo se vuelve "ciego al objeto [objektblind]" que se presenta como existente en la actitud natural, o incluso ciego al mundo, cuando la epojé se practica con respecto a éste (Hua VIII, 111). Por el contrario, destaca Husserl, "sigo viendo todo [für alles bleibe ich sehend]" (Hua VIII, 111. Trad. mía), si bien el carácter de existencia de las cosas y el mundo es puesto fuera de juego. En este orden de ideas, la reflexión fenomenológica, posibilitada por la epojé, puede verse como un ejercicio de tematización de las propias experiencias que les inhibe a éstas el carácter de existencia en el que aún se apoya la reflexión psicológica. Si seguimos esta caracterización husserliana de la epojé, no resulta acertado concebir las vivencias reflejadas como objetos cósicos que se interponen entre el fenomenológo y las cosas (o el mundo). Las vivencias reflejadas no son observadas, no marchan como cosas ante un observador, sino que, por el contrario, son oportunidades para investigar temáticamente la relacionalidad intrínseca entre la conciencia y el mundo.

Ocasionalmente, Husserl destaca el tema fenomenológico del tema, de una manera convergente con las ideas que aquí he desarrollado. Así, en un frag-

\footnotetext{
12 "It is not true, he [Husserl] writes, that, while reflecting on an act of perception, I become blind to the perceived object. In fact, during reflection 'I remain clearsighted for everything' (Husserl 1959, p. 111)." (Petitmengin \& Bitbol 2011, 27. Énfasis en el original.)
} 
mento de 1926, titulado "Thema und Epoche. [...]", menciona un corte (Schnitt) que consiste en pasar de considerar la subjetividad tematizante, en el marco de la reflexión trascendental, a considerar, en el marco de la actitud natural, el mundo como tema de investigación, y dentro de él a la subjetividad 'real', es decir, a la subjetividad concebida como parte del mundo real-natural (Naturreales). Este último es "inevitablemente [unweigerlich]" un "co-tema [Mitthema]" cuando consideramos el mundo en el marco de la actitud natural. ${ }^{13}$ Lo anterior sugiere que si entendemos la objetivación reflexiva en términos cósicos, como un proceso a través del cual las experiencias y la vida subjetiva se convierten en 'cosas' de cierto tipo, hay razones para suponer que el mundo real-natural permanece siendo aún un co-tema de nuestra consideración. La consecuencia es clara: entender la objetivación reflexiva en términos de cosificación es permanecer aún dentro de los límites de la actitud natural, e ignorar el carácter distintivo de la reflexión trascendental.

Una manera de capturar este carácter distintivo es a través de la distinción entre una objetualidad cósica y una objetualidad temática. La unidad de una vivencia reflejada no es una unidad cósica, caracterizada por una trascendencia análoga a la que tienen los objetos perceptuales, sino una unidad temática, fijada temporalmente por quien reflexiona (cf. Hua VIII, 99). De este modo, las vivencias en la reflexión fenomenológica no se presentan, en lo fundamental, como cosas o procesos, sino como temas interrelacionados que pueden expandirse o contraerse de acuerdo con el interés de quien reflexiona, en el marco de un "universo temático" (Hua VIII, 100).

\section{OBSERVACIONES FINALES}

Podría quizás objetarse que la noción de objetualidad temática es demasiado minimalista. En cierto sentido, comparto el espíritu de esta observación,

\footnotetext{
13 "Das transzendentale Leben wird dann selbst wieder zum Thema transzendentaler Reflexion - oder kann es werden, und in infinitum. Aber all diese Reflexionen sind immer wieder transzendental. Ich nehme als Thema die Subjektivität, als wie sie lebt und in ihrem Leben Gegenständlichkeiten jeder Art, Universa, Welten thematisch hat. Wenn sie sich selbst, als irgend lebend, zum Thema hat, so kann sie weiter reflektierend abermals dieses Thematisch-Haben zum Thema machen und sich als dieselbe erkennen, die vordem anderes zum Thema hatte oder schon sich selbst als in gewisser Weise lebend zum Thema hatte. Aber hier ist eben schon und immer wieder rein Subjektives Thema, und ins Unendliche wird Thema die Subjektivität in allen Reflexionen. Demgegenüber, ein Schnitt ist: nicht die thematisierende Subjektivität als Thema haben, sondern die Welt als Thema haben und darin die "reale" Subjektivität als theoretisches Thema, wobei Naturreales unweigerlich Mitthema ist." (Hua XXXIV, 12. Énfasis en el original)
} 
aunque no veo por qué sería una crítica a la reflexión fenomenológica, a menos que se le apliquen de antemano estándares de objetualidad que no le son propios. Un objeto temático carece de la trascendencia propia de un objeto perceptual, pero posee una cierta unidad, que permite distinguirlo de otros objetos temáticos. La unidad de un objeto temático, sin embargo, no depende de características intrínsecas de dicho objeto. Son el interés y la atención los que determinan los contornos cambiantes de un objeto temático, y no la existencia, durante la reflexión o previa a ella, de un objeto con características intrínsecas y al que, quien reflexiona, dirigiría la atención con la pretensión de capturarlas. Por lo demás, esta postura sobre el tipo de objetualidad característica de la reflexión fenomenológica es consistente con la ya mencionada afirmación heideggeriana de que la vida no tiene, ni puede tener el carácter de lo objetual. ${ }^{14}$

La crítica de la crítica de Heidegger a la reflexión fenomenológica desarrollada en el presente artículo, no agota los posibles escenarios de contraste entre la fenomenología hermenéutica y la fenomenología reflexiva. Sin embargo, es un escenario fundamental de dicho contraste, ya que la reflexión es una de las claves fenomenológicas ineludibles para valorar la confrontación de Heidegger con la fenomenología de Husserl. Antes de concluir, no está de más destacar que, sin lugar a dudas, hay mucho más en la fenomenología hermenéutica heideggeriana, en términos de contenido y de método, que su crítica a la reflexión husserliana. Por lo que se refiere a la problemática de la reflexión, sin embargo, es dudoso que una aprehensión positiva más extensa de la fenomenología de Heidegger resulte decisiva para dilucidar el componente específicamente crítico de su propuesta con respecto a la reflexión fenomenológica. Del mismo modo, sobra decir que el argumento que aquí he presentado no es suficiente para mostrar, en un sentido positivo, que la fenomenología reflexiva y la fenomenología hermenéutica son metodológicamente compatibles. Pero lo dicho hasta ahora respalda la idea de que ambas no son metodológicamente incompatibles, si la razón para sustentar la oposición e incompatibilidad entre ambas es el uso de la reflexión (von Herrmann 2000; Adrián 2005, 2010). Estas consideraciones abren la posibilidad de explorar las relaciones entre la fenomenología reflexiva y la fenomenología hermenéutica sobre un suelo metodológico que no está minado de antemano.

14 "[...] das Leben is kein Objekt und kann nie Objekt werden: es ist nichts Objektartiges." (GA 58, 236) 


\section{REFERENCIAS BIBLIOGRÁFICAS}

Adrián, J. (2005) "Hermeneutische versus reflexive Phänomenologie.", En: Tymieniecka, A. (Ed.). Logos of Phenomenology and Phenomenology of Logos. Book One: Phenomenology as the Critique of Reason in Contemporary Criticism and Interpretation. Dordrecht: Springer.

Adrián, J. (2010) Heidegger y la genealogía de la pregunta por el sentido del ser. Una articulación temática y metodológica de su obra temprana. Herder: Barcelona.

Cai, W. (2011) Reflection As a Form of Human Life: Methodological Issues in Phenomenology. Copenhagen: Press of the Faculty of Humanities, University of Copenhagen.

Crowell, S. (2001) Husserl, Heidegger and the Space of Meaning. Evanston: Northwestern University Press.

Heidegger, M. (1979) [= GA 20] Prolegomena zur Geschichte des Zeitsbegriffs. Hrsg. von Petra Jaeger. Frankfurt a. M.: Vittorio Klostermann.

- (1988) [= GA 63] Ontologie. Hermeneutik der Faktizität. Hrsg. von K. BrökerOltmanns. Frankfurt a. M.: Vittorio Klostermann.

- (1993) [= GA 58] Grundprobleme der Phänomenologie (1919-1920). Hrsg. von H.-H. Gander. Frankfurt a. M.: Vittorio Klostermann.

- (2005) La idea de la filosofía y el problema de la concepción de mundo. Trad. J. Adrián Escudero. Madrid: Herder.

- (1999) [= GA 56/57] Zur Bestimmung der Philosophie. Frankfurt a. M.: Vittorio Klostermann.

- (2006) Prolegómenos para una Historia del Concepto de Tiempo. Trad. Jaime Aspiunza. Madrid: Alianza Editorial.

Husserl, E. (1949) Ideas relativas a una fenomenología pura y una filosofía fenomenológica. Primer libro: Introducción general a la fenomenología pura. Trad. José Gaos. México: Fondo de Cultura Económica.

- (1959) [= Hua VIII] Erste Philosophie. Zweiter Teil. Hrsg. von R. Boehm. Den Haag: Martinus Nijhoff.

- (1973) [Hua I] Cartesianische Meditationen und Pariser Vorträge. Hrsg. von S. Strasser. Den Haag: Martinus Nijhoff.

- (1976) [= Hua III/1] Ideen zu einer reinen Phänomenologie und phänomenologischen Philosophie. Erstes Buch: Allgemeine Einführung in die reine Phänomenologie. Hrsg. von Karl Schuhmann. Den Haag: Martinus Nijhoff.

- (2002) [= Hua XXXIV] Zur Phänomenologischen Reduktion. Texte aus dem Nachlass (1926 - 1935). Hrsg. von S. Luft. Dordrecht: Kluwer Academic Publishers.

- (2005) Meditaciones cartesianas. Trad. José Gaos y Miguel García Baró. México: Fondo de Cultura Económica.

Natorp, P. (1912) Allgemeine Psychologie nach kritischer Methode. Tübingen: Mohr-Siebeck.

Petitmengin, C. \& Bitbol, M. (2011) "On Pure Reflection. A Reply to Dan Zahavi". En: Journal of Consciousness Studies, 18, (2): 24-37.

von Herrmann, F.-W. (2000) Hermeneutik und Reflexion. Der Begriff der Phänomenologie bei Heidegger und Husserl. Frankfurt a. M.: Vittorio Klostermann.

Zahavi, D. (1999) Self-Awareness and Alterity: A Phenomenological Investigation. Evanston: Northwestern University Press.

- (2003a) "How to investigate subjectivity? Natorp and Heidegger on reflection." En: Continental Philosophy Review 36: 155-176.

- (2003b) Husserl's Phenomenology. Stanford: Stanford University Press. 
- (2005). Subjectivity and Selfhood. Investigating the First Person Perspective. Cambridge: MIT Press.

- (2009) "Phänomenologie und Transzendentalphilosophie." En: Figal, G. \& Gander H.-H. (Hrsg.). Husserl und Heidegger. Neue Perspektiven. Frankfurt a. M.: Vittorio Klostermann. 